\title{
Timing and Interval Effects of Repeated Inseminations by Roosters on the Fathering of Chicks
}

\author{
Hoang Yen T. Bui ${ }^{1}$, Yuki Nakamura ${ }^{1}$, Atsushi Takenouchi ${ }^{1}$, Masaoki Tsudzuki ${ }^{1,2}$ and Teruo Maeda ${ }^{1,2}$ \\ ${ }^{1}$ Graduate School of Biosphere Science, Hiroshima University, Higashi-Hiroshima 739-8528, Japan \\ ${ }^{2}$ Japanese Avian Bioresource Project Center, Hiroshima University, Higashi-Hiroshima 739-8528, Japan
}

\begin{abstract}
The present study investigated the effects of timing and intervals between repeated inseminations by different rooster breeds on the fathering of chicks. The semen from Barred Plymouth Rock (BP), Japanese Silkie White (JS), and White Plymouth Rock (WP) males were used. Eighteen hens (7-month-old BP) were divided into four Groups $(A-D)$. In all groups, the hens were first inseminated with the semen from BP males at $3 \mathrm{pm}$ on day 0 . The hens in Group A $(n=3)$ were inseminated with the semen from JS and WP males at $3 \mathrm{pm}$ on days 5 and 10, respectively. Those in Group B $(n=3)$ were inseminated with the semen from JS and WP males at $3 \mathrm{pm}$ on days 5 and 15 , respectively. The hens in Group C $(n=6)$ were inseminated with the semen from JS and WP males at 6 am on days 5 and 10, respectively. Lastly, those in Group D $(n=6)$ were inseminated with the semen from JS and WP males at 6 am on days 10 and 20, respectively. The chicks obtained were classified on the basis of their phenotype matching with the breeding male phenotype. The majority of chicks $(82.4 \%$ to $100 \%$ in Groups A and B) were fathered by the sperm from the subsequent insemination when repeated insemination was performed in the afternoon. Moreover, the chicks produced by the most recent insemination in the groups that were subjected to morning insemination could be obtained the next day after insemination (Groups $\mathrm{C}$ and $\mathrm{D}$ ). The percentage of chicks fathered by the sperm from the subsequent insemination was $95.2 \%$ to $100 \%$ when the inter-insemination interval was 10 days (Groups B and D); these percentages decreased when the duration of the interval between inseminations was decreased to 5 days in Groups A, B, and C.
\end{abstract}

Key words: fertilization, interval, repeated insemination, sperm storage tubules, timing

J. Poult. Sci., 55: 301-306, 2018

\section{Introduction}

In poultry, fertilization usually occurs 1 to 3 days after insemination, and it is maintained for several weeks (Johnson, 1986a). Sperm transit through the sperm storage tubules (SSTs) located in the uterovaginal junctions and infundibulums of hens, and are gradually used for fertilization (Brillard, 1993; Bakst, 1994). However, fertility is reduced by 1 week after insemination, particularly when the artificial insemination (AI) method is used (Johnson, 1986a). Therefore, repeated inseminations are necessary to maintain fertility. Consequently, the sperm populations from different inseminations compete to access the SSTs, and the sperm from the last insemination are predominantly responsible for fathering chicks (Compton et al., 1978; Bakst, 1994). Several studies have explained the mechanism of sperm competi-

Received: December 15, 2017, Accepted: March 3, 2018

Released Online Advance Publication: May 25, 2018

Correspondence: T. Maeda, Graduate School of Biosphere Science, Hiroshima University, Higashi-Hiroshima 739-8528, Japan.

(E-mail: temaeda@hiroshima-u.ac.jp) tion in the SSTs during tandem inseminations; however, the information obtained has been inconsistent. Compton et al. (1978) and Christensen (1981) suggested that the precedence of sperm from the previous insemination was due to the stratification or displacement of sperm in the SSTs. In contrast, Birkhead and Parker (1997) reported that the sperm from tandem insemination were mixed in the SSTs, and loss of sperm from the previous insemination by the time of the subsequent insemination resulted in fertilization dominance of the most recent sperm. This contradiction between studies may be the result of inconsistent experimental factors, such as timing and intervals between inseminations. In the present study, we examined the effects of the timing of repeated insemination with respect to oviposition or ovulation (early morning versus afternoon) on the proportion of chicks produced from each insemination. We also investigated the effects of different intervals ( 5 days versus 10 days) between inseminations on the timing of the day of appearance of the chicks from each insemination. 


\section{Materials and Methods}

\section{Animals}

Semen was collected for AI from the males of three breed phenotypes: 7-month-old Barred Plymouth Rock (BP), 2year-old White Plymouth Rock (WP), and 1-year-old Japanese Silkie White (JS). The semen was used to inseminate 7month-old BP hens. The roosters and hens of the BP breed had both black and white plumage, yellow legs, and four toes. The WP roosters had white plumage, yellow legs, and single combs. The JS roosters had white plumage, black legs, and five toes. All of the birds were caged individually and maintained in environmentally controlled houses on a 14 $\mathrm{h}$ light: $10 \mathrm{~h}$ dark photoperiod. Feed and water were provided ad libitum. All animals used in the present study were handled in accordance with the regulations of the Animal Experiment Committee of Hiroshima University.

\section{Semen Collection and AI}

Semen samples were collected using the abdominal massage method of Burrows and Quinn (1937). Semen from at least two roosters of each breed were pooled to avoid the effects of individual males on fertilizing ability. After semen collection, $100 \mu l$ of pooled semen was immediately placed into the vaginal orifice of each hen at a depth of $2 \mathrm{~cm}$ following a previously described method (Quinn and Burrows, 1936). The number of sperm per AI in each group was measured using a hemocytometer after insemination to confirm whether enough sperm had been deposited.

\section{Experimental Design}

In the domestic hen, oviposition and ovulation usually occur in the morning (Johnson, 1986b). It has been reported that fertility following inseminations in the afternoon is higher than that observed after morning insemination (Bornstein et al., 1960). One of objectives of the present study was to elucidate whether repeated inseminations with sperm could result in fertilization within several hours. Therefore, insemination timing was set for the early morning $(6 \mathrm{am})$ or the afternoon $(3 \mathrm{pm})$. The insemination interval was also designed as 5 or 10 days, because it has been stated that hen remain fertile for as long as 5-6 days after insemination, and then fertility declines rapidly (Johnson, 1986a).

Hens were tandemly inseminated following different schedules based on grouping. Eighteen hens were randomly assigned to four groups: three hens each in Groups A and B, and six hens each in Groups C and D. The experimental design is shown in Figure 1. In all the groups, the hens were first inseminated with the semen from BP males at $3 \mathrm{pm}$ on day 0. Those in Group A were inseminated with the semen from JS and WP males at $3 \mathrm{pm}$ on days 5 and 10 , respectively. The hens in Group B were inseminated with the semen from JS and WP males at $3 \mathrm{pm}$ on days 5 and 15 , respectively. Those in Group $\mathrm{C}$ were inseminated with the semen from JS and WP males at 6 am on days 5 and 10 , respectively. Lastly, the hens in Group D were inseminated with the semen from JS males and WP males at 6 am on days 10 and 20 , respectively.

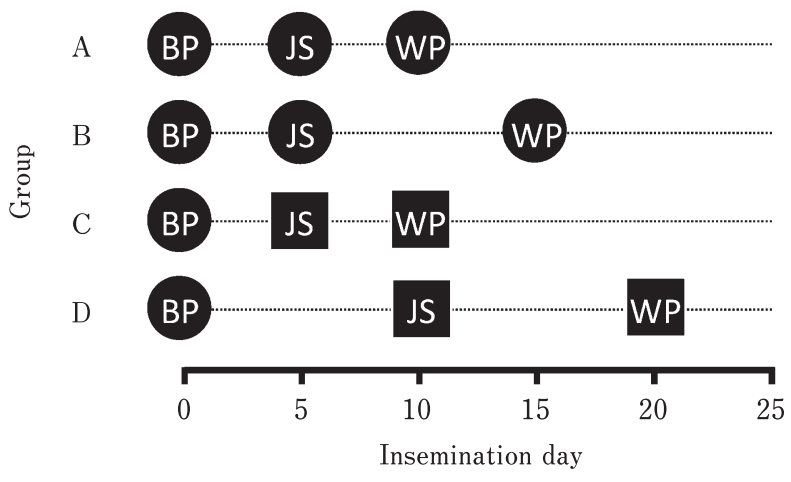

Fig. 1. The artificial insemination schedule.

BP indicates insemination using semen from Barred Plymouth Rock males at $3 \mathrm{pm}$.

(s) indicates insemination using semen from Japanese Silkie White males at $3 \mathrm{pm}$.

Js indicates insemination using semen from Japanese Silkie White males at 6 am.

(UP indicates insemination using semen from White Plymouth Rock males at $3 \mathrm{pm}$.

WP indicates insemination using semen from White Plymouth Rock males at 6 am.

\section{Egg Collection and Incubation}

The eggs from each group were collected daily until 21 days after the last insemination and were then preserved at $15^{\circ} \mathrm{C}$ and $60 \%$ humidity. The eggs from each 7-day egg collection were hatched in a Zenkei incubator (Zenkeien Factory, Miyakoda, Japan). The incubation conditions were $37.8^{\circ} \mathrm{C}, 60 \%$ humidity, and an automatic 1-hour interval rotation.

\section{Distinction of the Origin of Progeny Based on the Pheno- type of the Chicks}

The chicks were classified based on the phenotypic characteristics of the feathers, legs, and toes. The BP semen generated chicks with both black and white feathers, yellow legs, and four toes. The WP semen generated chicks with white feathers and four toes. The JS semen generated chicks with a mulberry crown, black legs and beaks, and five toes.

\section{Evaluation}

The day of appearance of chicks was assessed from the insemination day (which was defined as day 0 ). The duration of the period till the appearance of the chicks was defined as the first day after insemination to the day of appearance of the chick. Therefore, the periods in each group were as follows: Group A: 2-6, 7-11, and 12-31 days; Group B: 2-6, 7-16, and 17-36 days; Group C: 2-6, 7-11, and 12-31 days; and Group D: 2-11, 12-21, and 22-41 days. The appearance rate of chicks was calculated as the number of that type of chick obtained per total number of hens inseminated. The percentage of chicks produced by each male breed was evaluated using the following formula:

$\%$ of chicks $=$

total number of chicks of that male breed total number of chicks obtained 
Table 1. The chicks obtained from different male phenotypes, according to variations in the time of insemination and the interval between inseminations

\begin{tabular}{|c|c|c|c|c|c|c|c|c|c|c|c|c|c|c|c|c|c|c|c|c|c|c|}
\hline \multirow{2}{*}{ Group } & \multirow{2}{*}{$\begin{array}{l}\text { Hen } \\
\text { No. }\end{array}$} & \multicolumn{21}{|c|}{ Days after artificial insemination } \\
\hline & & 0 & 1 & 2 & 3 & 4 & 5 & 6 & 7 & 8 & 9 & 10 & 11 & 12 & 13 & 14 & 15 & 16 & 17 & 18 & 19 & 20 \\
\hline \multirow{4}{*}{ A } & & BP & & & & & IS & & & & & WP & & & & & & & & & & \\
\hline & 1 & & & & & BP & $\mathrm{BP}$ & $\mathrm{BP}$ & & JS & JS & & JS & & WP & & JS & WP & & & & \\
\hline & 2 & & & $\mathrm{BP}$ & & BP & $\mathrm{BP}$ & & JS & JS & & JS & JS & & WP & WP & & WP & WP & WP & & \\
\hline & 3 & & & BP & $\mathrm{BP}$ & BP & & $\mathrm{BP}$ & JS & & JS & JS & & & JS & WP & & & JS & WP & WP & - \\
\hline \multirow{4}{*}{ B } & & BP & & & & & (Is) & & & & & & & & & & WP & & & & & \\
\hline & 8 & & & & BP & BP & & BP & JS & & JS & JS & JS & & JS & JS & & JS & $\times$ & & WP & \\
\hline & 9 & & & & & & BP & & JS & JS & & JS & JS & & BP & & JS & & WP & WP & & \\
\hline & 13 & & & BP & BP & BP & $\mathrm{BP}$ & & JS & $\times$ & BP & JS & & & $\mathrm{BP}$ & & JS & $\times$ & WP & & WP & \\
\hline \multirow{7}{*}{$\mathrm{C}$} & & BP & & & & & JS & & & & & WP & & & & & & & & & & \\
\hline & 14 & & & & BP & & $\mathrm{BP}$ & JS & BP & $\mathrm{BP}$ & JS & & JS & $\times$ & & WP & WP & WP & WP & $\times$ & WP & $x$ \\
\hline & 15 & & & BP & - & & BP & BP & & $\mathrm{BP}$ & JS & JS & WP & & WP & WP & WP & WP & & WP & WP & WP \\
\hline & 16 & & & BP & BP & BP & BP & BP & BP & JS & BP & & JS & & WP & WP & WP & $\times$ & & WP & WP & WP \\
\hline & 17 & & & & & $\mathrm{BP}$ & & & & & & & JS & & & WP & & & & JS & & WP \\
\hline & 18 & & & & BP & & BP & & BP & & & & & & & & & & & & & WP \\
\hline & 19 & & & BP & BP & & $\mathrm{BP}$ & $\mathrm{BP}$ & $\mathrm{BP}$ & & $\mathrm{BP}$ & & BP & & WP & WP & & WP & WP & & WP & WP \\
\hline \multirow{7}{*}{$\mathrm{D}$} & & BP & & & & & & & & & & JS & & & & & & & & & & WP \\
\hline & 4 & & & & BP & & & BP & BP & & BP & BP & & JS & JS & & JS & JS & JS & & JS & JS \\
\hline & 5 & & & BP & & & & BP & BP & & BP & BP & JS & & JS & JS & & JS & & JS & & $\times$ \\
\hline & 6 & & & & & BP & & & & & & BP & & BP & & & & & & & & \\
\hline & 7 & & & BP & BP & BP & & BP & BP & & BP & BP & & JS & JS & & JS & JS & & & JS & JS \\
\hline & 10 & & & BP & BP & BP & $\times$ & & & & $\times$ & & - & & JS & $\times$ & & & $x$ & JS & & \\
\hline & 11 & & & & BP & BP & BP & BP & $\mathrm{BP}$ & & BP & & $\times$ & & JS & & & & & & & \\
\hline \multirow{2}{*}{ Group } & Hen & \multicolumn{21}{|c|}{ Days after artificial insemination } \\
\hline & No. & 21 & 22 & 23 & 24 & 25 & 26 & 27 & 28 & 29 & 30 & 31 & 32 & 33 & 34 & 35 & 36 & 37 & 38 & 39 & 40 & 41 \\
\hline \multirow{3}{*}{ A } & 1 & & & & & $\times$ & & - & - & - & - & - & & & & & & & & & & \\
\hline & 2 & WP & & WP & WP & - & & WP & - & - & - & - & & & & & & & & & & \\
\hline & 3 & - & & - & - & - & & & - & - & - & & & & & & & & & & & \\
\hline \multirow{3}{*}{ B } & 8 & WP & WP & WP & & WP & WP & & WP & - & & - & & - & - & & & & & & & \\
\hline & 9 & WP & & WP & WP & & WP & & - & - & & - & - & & - & & & & & & & \\
\hline & 13 & WP & WP & WP & WP & WP & & - & - & - & & - & - & - & - & & - & & & & & \\
\hline \multirow{6}{*}{$\mathrm{C}$} & 14 & $\times$ & WP & - & & & & & - & - & & & & & & & & & & & & \\
\hline & 15 & WP & WP & & - & WP & - & - & & WP & - & & & & & & & & & & & \\
\hline & 16 & WP & & - & WP & $\times$ & WP & - & & - & - & - & & & & & & & & & & \\
\hline & 17 & & BP & & - & WP & - & & - & - & - & & & & & & & & & & & \\
\hline & 18 & & & & & & & & & & & & & & & & & & & & & \\
\hline & 19 & & & - & & - & - & & - & - & - & & & & & & & & & & & \\
\hline \multirow{6}{*}{ D } & 4 & JS & & - & & - & & - & 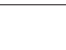 & - & - & & - & - & - & & & - & - & & - & - \\
\hline & 5 & JS & & WP & WP & $\times$ & & - & - & - & - & & - & - & - & & & - & - & - & - & - \\
\hline & 6 & & & & & WP & WP & & WP & WP & & & - & & - & & & & & & & \\
\hline & 7 & & & WP & & WP & WP & & JS & WP & & WP & WP & - & WP & & & - & - & & - & - \\
\hline & 10 & JS & & WP & WP & & & WP & & $\times$ & WP & & WP & - & - & & & - & & & & - \\
\hline & 11 & & & WP & & & WP & & & & & & & & & & & & & & & \\
\hline
\end{tabular}

indicates insemination using semen from Barred Plymouth Rock (BP) males at $3 \mathrm{pm}$ 
Table 2. The appearance of the offspring obtained after artificial insemination

\begin{tabular}{|c|c|c|c|c|c|c|c|c|c|c|c|c|}
\hline \multirow{2}{*}{$\begin{array}{l}\text { Group } \\
\text { Breed }\end{array}$} & \multicolumn{3}{|c|}{ A } & \multicolumn{3}{|c|}{$\mathrm{B}$} & \multicolumn{3}{|c|}{$\mathrm{C}$} & \multicolumn{3}{|c|}{$\mathrm{D}$} \\
\hline & $\mathrm{BP}$ & JS & WP & $\mathrm{BP}$ & JS & WP & BP & JS & WP & $\mathrm{BP}$ & JS & WP \\
\hline $\begin{array}{l}\text { Interval between } \\
\text { inseminations (days) }\end{array}$ & \multicolumn{2}{|c|}{5} & 5 & \multicolumn{2}{|c|}{5} & 10 & \multicolumn{2}{|c|}{5} & 5 & \multicolumn{2}{|c|}{10} & 10 \\
\hline Earliest appearance day ${ }^{1}$ & 2 & 2 & 3 & 2 & 2 & 2 & 2 & 1 & 1 & 2 & 1 & 3 \\
\hline Last appearance day ${ }^{1}$ & 6 & 12 & 17 & 13 & 11 & 13 & 22 & 13 & 19 & 12 & 18 & 14 \\
\hline Period of appearance ${ }^{2}$ & 5 & 11 & 15 & 12 & 10 & 12 & 21 & 13 & 19 & 11 & 18 & 12 \\
\hline
\end{tabular}

${ }^{1}$ The day of appearance of chicks was assessed from the day of insemination, which was designated as Day 0 .

${ }^{2}$ The period of appearance of chicks (days) was calculated from the earliest to the last day of the appearance of that type of chick. BP: Barred Plymouth Rock; JS: Japanese Silkie White; WP: White Plymouth Rock.

Groups A, B, C, and D comprised 7-month-old BP hens ( $n=3,3,6$, and 6 in Groups A, B, C, and D, respectively).

\section{Results}

The number of sperm per insemination was greater than 80 million. The timings of the appearances of the chicks obtained after AI with semen from the three male breeds are shown in Tables 1 and 2. The earliest appearance of fertilized eggs was most commonly 2 days after insemination in all experimental groups for both the first and repeated inseminations. However, in the groups that underwent repeated insemination in the morning (Groups $\mathrm{C}$ and $\mathrm{D}$ ), the fertilized eggs occasionally appeared 1 day after the second and third inseminations. The last day of appearance of chicks generated by BP males was $6,13,22$, and 12 days after the insemination day in Group A, B, C, and D, respectively. The chicks from each male breed phenotype appeared for at least 5 days after the insemination day. The longest period of appearance was 21 days in Group C (BP chicks).

Figure 2 shows the changing rate of chicks obtained from different males after repeat insemination at different times of the day and with different intervals between inseminations. The chicks generated from the previous insemination continued to appear after the subsequent insemination. The percentages of chicks generated from the most recent insemination were $100 \%$ and $82.4 \%$ in Group A, $83.3 \%$ in Group B (second insemination performed in the afternoon with an interval of 5 days between inseminations), and $41.2 \%$ and 94.7\% in Group C (hens inseminated in the morning with an interval of 5 days between inseminations). In addition, the percentage of chicks generated by the sperm from the most recent insemination was increased both when the interval between inseminations was 10 days in Group D (95.8\% and $95.2 \%$ ), and when there was a 10-day interval between the second and third inseminations in Group B $(100 \%)$.

\section{Discussion}

It has been reported that insemination with $80-90$ million spermatozoa per insemination is generally considered adequate to ensure high fertility in fowl (Johnson, 1986a). In the present study, the number of sperm per insemination was greater than 80 million. Good fertility was observed for as long as 1 week after insemination. Therefore, it was considered that all AI in the present study was performed appropriately.

In birds, fertilized eggs and chicks can reportedly be obtained approximately 2 to 3 weeks after insemination due to the existence of SSTs in the reproductive tract (Bakst, 1994). Similarly, our results showed that the period from insemination to the appearance of chicks was 5 to 21 days. After insemination, sperm can enter and survive in the SSTs, after which sperm are gradually used for fertilization for several weeks (Brillard, 1993; Bakst, 1994). Hence, when repeated inseminations are performed, chicks produced by the previous insemination are still observed (Cheng et al., 1983). Consequently, the interval between inseminations influences both the sperm competition and the rate of progeny obtained from the different males (Birkhead, 1998; Ma et al., 2013). Our results showed that offspring from different males could be obtained; however, the highest percentage of progeny was fathered by sperm from the most recent insemination. In addition, the appearance of chicks generated by the sperm from the previous and subsequent inseminations was simultaneous and did not depend on the interval between inseminations. Compton et al. (1978) suggested that the sperm from the last insemination took precedence due to the stratification or displacement of sperm in the SSTs after repeat insemination. Thus, the offspring generated by semen from previous inseminations would be absent or would only appear after the sperm from the subsequent insemination were depleted because of the stratification of sperm (Christensen, 1981). However, the simultaneous appearance of the different types of chicks in the present study might be due to the mixing of sperm from tandem inseminations in the SSTs (Birkhead and Parker, 1997). We also found that the appearance of chicks generated by the most recent insemination increased when the duration of the interval between inseminations was increased. This might be due to the renewed sperm in the SSTs by the subsequent insemination fertilizing the majority of the egg dominantly. Thus, when the interval between inseminations was increased, there was a higher proportion of chicks generated by the sperm from the most recent insemination (Birkhead, 

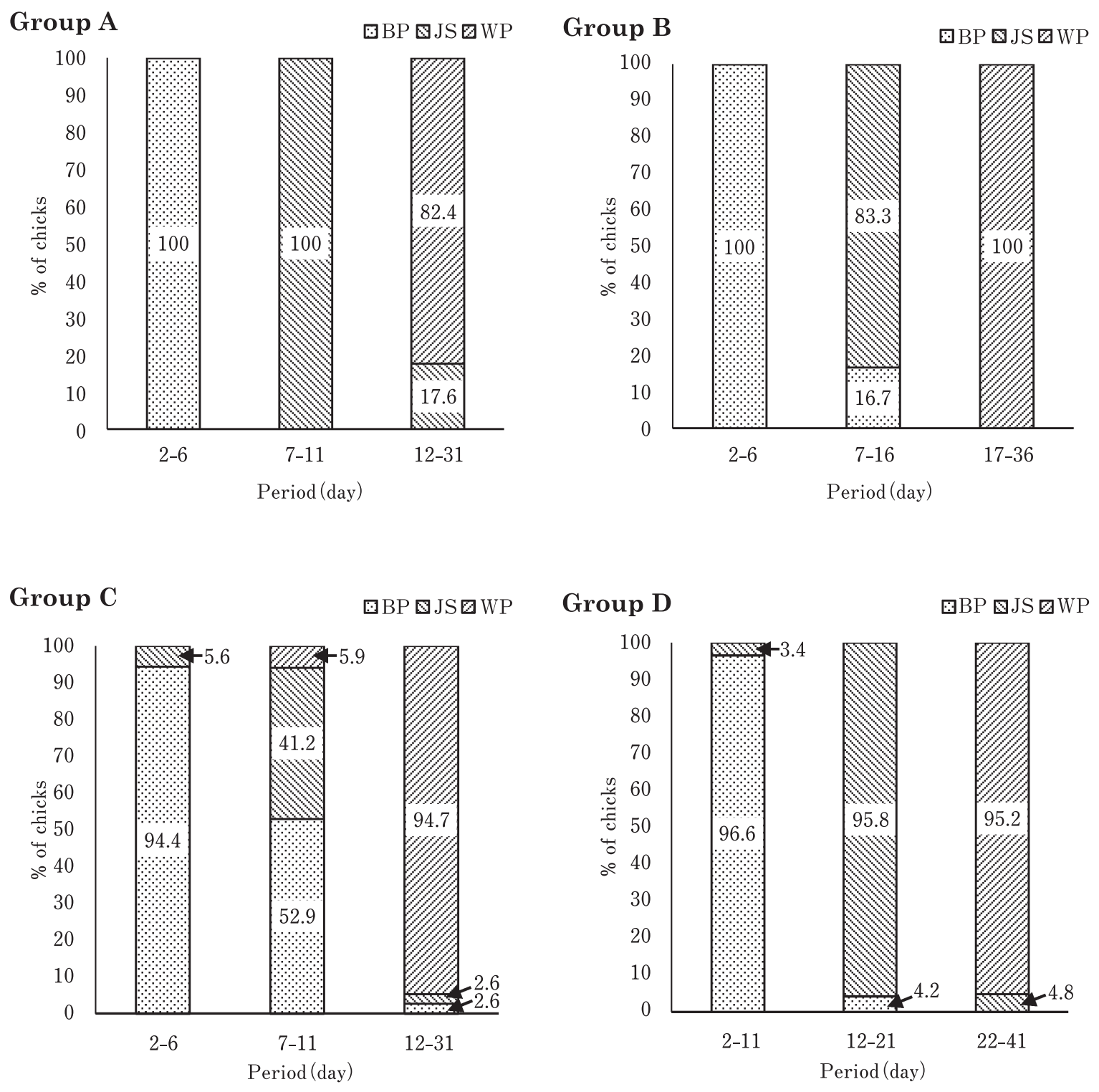

Fig. 2. The percentage of offspring obtained that were fathered by each male breed, based on varying intervals between inseminations. The duration of the period till the appearance of the chicks was defined as the first day after insemination to the day of appearance of the chick. BP: Barred Plymouth Rock; JS: Japanese Silkie White; WP: White Plymouth Rock.

1998).

The timing of insemination reportedly affects the fertilization rate in chickens (Mian et al., 1991). Thus, this factor may affect the timing of the appearance of chicks generated by sperm from different males after repeated insemination (Birkhead, 1998). In the present study, we examined the effects of the time of insemination on the timing of the appearance of offspring by performing repeated inseminations in the early morning (6 am) or afternoon ( $3 \mathrm{pm})$ (Bhatti, 1987). Our results showed that eggs fertilized by the sub- sequent insemination could be collected 1 day after insemination when hens were inseminated in the early morning, whereas this period was 2 days after repeated insemination performed in the afternoon. In poultry, the offspring can be obtained generally 1 day (Cheng et al., 1983) and 2 days (Ma et al., 2013) after insemination, depending on the timing of insemination. Ovulation reportedly occurs 15 minutes after oviposition, and each ovum spends only 15-30 minutes in the infundibulum for fertilization (Ogawa et al., 1996). In addition, the time required for sperm to move to the fertilization 
site is approximately 1 hour after insemination (Johnson, 1986a). Thus, when insemination is performed during oviposition, sperm can reach the infundibulum to fertilize the ovum before or just after ovulation of the next ovum, even if the female retained sperm from another insemination (Cheng et al., 1983). In contrast, when hens are inseminated in the afternoon, the next ovum has already been ovulated and transferred out of the fertilization section of the reproductive tract, and therefore sperm cannot fertilize that ovum (Bhatti, 1987). After insemination, chicken sperm reach and stay in the SSTs for 1 to 3 days after insemination (Brillard, 1993), and are then released to fertilize the ovum released the next day. Ito et al. (2011) reported that progesterone is a spermreleasing factor from the SSTs in birds. Therefore, the sperm stored in SSTs are released by progesterone and are then fertilized. The fertilized eggs can be collected 2 days after insemination.

In conclusion, we found that chicks can be obtained for approximately 3 weeks after insemination due to the retention of sperm in the SSTs. In repeated insemination, chicks produced by the sperm from tandem inseminations appeared simultaneously due to the mixing of sperm in the SSTs. The majority of obtained chicks were generated by sperm from the most recent insemination due to the loss of sperm from the previous insemination in the SSTs. As a result, the rate of obtained chicks generated by sperm from the following insemination increased when the interval time between inseminations was increased from 5 to 10 days. The day of appearance of chicks generated by sperm from the most recent insemination was 1 day after insemination when hens were inseminated in the early morning.

\section{Acknowledgments}

We thank Kelly Zammit, BVSc, from Edanz Group (www. edanzediting.com/ac), for editing a draft of this manuscript. We also thank Professor Joseph James Lauer (Institute for Foreign Language Research and Education, Hiroshima University, Higashi-Hiroshima, Japan) for the language editing.

\section{References}

Bakst MR. Oviducal sperm selection, transport, and storage in poultry. Poultry Science Reviews, 5: 117-143. 1994.
Bhatti BM. Exogenous regulation of the time of oviposition in the domestic fowl. World's Poultry Science Journal, 43: 116-131. 1987.

Birkhead TR. Sperm competition in birds. Reviews of Reproduction, 3: 123-129. 1998.

Birkhead TR and Parker GA. Sperm competition and mating systems. In: Behavioral Ecology: An evolutionary approach (Krebs JR and Davies NB eds.). $4^{\text {th }}$ ed . pp. 121-145. Oxford: Blackwell. 1997.

Bornstein S, Schindler H, Gabriel I and Moses E. Fertilization rate of chickens inseminated in the morning or in the afternoon. Ktavim, 10: 183-191. 1960.

Brillard JP. Sperm storage and transport following natural mating and artificial insemination. Poultry Science, 72: 923-928. 1993.

Burrows WH and Quinn JP. The collection of spermatozoa from the domestic fowl and turkey. Poultry Science, 16: 19-24. 1937.

Cheng KM, Burns JT and McKinney F. Forced copulation in captive mallards III. sperm competition. The Auk, 100: 302-310. 1983.

Christensen VL. Effect of insemination intervals on oviducal sperm storage in turkeys. Poultry Science, 60: 2150-2156. 1981.

Compton MM, Van Krey HP and Siegel PB. The filling and emptying of the uterovaginal sperm-host glands in the domestic hen. Poultry Science, 57: 1696-1700. 1978.

Ito T, Yoshizaki N, Tokumoto T, Ono H, Yoshimura T, Tsukada A, Kansaku N and Sasanami T. Progesterone is a sperm-releasing factor from the sperm-storage tubules in birds. Endocrinology, 152: 3952-3962. 2011.

Johnson AL. Reproduction in the male. In: Avian Physiology (Sturkie PD ed.). pp. 432-451. Springer. New York. 1986a.

Johnson AL. Reproduction in the female. In: Avian Physiology (Sturkie PD ed.). pp. 403-431. Springer. New York. 1986b.

Ma J, Chen P, Xu G, Peng Z, Yang C, Shu D, Wang J, Luo C and Qu H. Sperm competition greatly decreases the time interval when breeder hens are artificially inseminated by different cockerels. The Journal of Applied Poultry Research, 22: 19-26. 2013.

Mian AA, Bhatti BM, Hussain A and Qureshi MS. Fertility of hens as affacted by the time of insemination. Pakistan Journal of Agricultural Research, 12: 222-225. 1991.

Ogawa $\mathrm{H}$, Kuwayama $\mathrm{T}$ and Tanaka $\mathrm{K}$. The timing of ovulation after oviposition and the time spent by the ovum in each portion of oviduct in the guinea fowl. Japanese Poultry Science, 33: 118-122. 1996.

Quinn JP and Burrows WH. Artificial insemination in flowls. Journal of Heredity, 27: 31-38. 1936. 